\title{
Modelling positive Granger Causality and negative phase lag between cortical areas
}

Fernanda S. Matias ${ }^{1,2}$, Leonardo L. Gollo ${ }^{3}$, Pedro V. Carelli ${ }^{1}$,

Steven L. Bressler ${ }^{4}$, Mauro Copelli ${ }^{1}$, and Claudio R. Mirasso ${ }^{2, \dagger}$

${ }^{1}$ Departamento de Física, Universidade Federal de

Pernambuco, Recife, PE 50670-901 Brazil

${ }^{2}$ Instituto de Fisica Interdisciplinar y Sistemas Complejos, CSIC-UIB, Campus Universitat de les Illes Balears E-07122

Palma de Mallorca, Spain

${ }^{3}$ Systems Neuroscience Group, Queensland Institute of Medical

Research, Brisbane, QLD 4006, Australia

${ }^{4}$ Center for Complex Systems and Brain Sciences, Dept. of

Psychology, Florida Atlantic University, Boca Raton, FL 33431

†claudio@ifisc.uib-csic.es 


\begin{abstract}
Different measures of directional influence have been employed to infer effective connectivity in the brain. When the connectivity between two regions is such that one them (the sender) strongly influences the other (the receiver), a positive phase lag is often expected. The assumption is that the time difference implicit in the relative phase reflects the transmission time of neuronal activity. However, Brovelli et al. (2004) observed that, in monkeys engaged in processing a cognitive task, a dominant directional influence from one area of sensorimotor cortex to another may be accompanied by either a negative or a positive time delay. Here we present a model of two brain regions, coupled with a well-defined directional influence, that displays similar features to those observed in the experimental data. This model is inspired by the theoretical framework of Anticipated Synchronization developed in the field of dynamical systems. Anticipated Synchronization is a form of synchronization that occurs when a unidirectional influence is transmitted from a sender to a receiver, but the receiver leads the sender in time. This counterintuitive synchronization regime can be a stable solution of two dynamical systems coupled in a masterslave (sender-receiver) configuration when the slave receives a negative delayed self-feedback. Despite efforts to understand the dynamics of Anticipated Synchronization, experimental evidence for it in the brain has been lacking. By reproducing experimental delay times and coherence spectra, our results provide a theoretical basis for the underlying mechanisms of the observed dynamics, and suggest that the primate cortex could operate in a regime of Anticipated Synchronization as part of normal neurocognitive function.
\end{abstract}

\title{
Introduction
}

Phase synchronization is extensively studied in the brain, where it has been hypothesized to underlie neurocognitive phenomena such as binding (Singer, 1999), temporal coding (Brette, 2012), spatial attention (Banerjee et al., 2011) and other higher cognitive functions (Wang, 2010) (see (Uhlhaas et al., 2009) for a recent review). Phase synchronization (Pikovsky et al., 2001) has been related to large-scale information integration (Varela et al., 2001), efficiency of information exchange (Fries, 2005), and both working and long-term memory (Fell and Axmacher, 2011). Correlation measures in the frequency domain are the most widely employed tools for measuring phase synchronization, which is typically used to infer interactions between brain areas (Siegel et al., 2012, Bressler and Menon, 2010). However, correlation alone cannot reveal the in- 
fluences that are exerted by neurons in one area on those in the other by axonal transmission and synaptic effects.

One approach to detecting directional influence in the brain has been to infer it from relative phase measures (Marsden et al., 2001; Williams et al., 2002; Schnitzler and Gross, 2005; Sauseng and Klimesch, 2008; Gregoriou et al., 2009) of neuroelectric indices, such as the electroencephalogram (EEG). The assumption here is that the timing difference implicit in relative phase reflects the transmission time of neural activity. By contrast, other measures of directional influence, such as Granger Causality (GC or G-causality), have emerged in recent years as an alternative approach that is grounded in the theoretical framework of statistical predictability between stochastic processes (Granger, 1969; Bressler and Seth, 2011). Alternative methods include partial directed coherence (Baccalá and Sameshima, 2001), nonlinear GC (Marinazzo et al., 2008; Marinazzo et al., 2011; He et al., 2014) and transfer entropy (Vicente et al., 2011; Lobier et al., 2014), among others (Pereda et al., 2005).

A dominant value for directional influence from one brain area $(\mathrm{A})$ to another (B) indicates that the activity of neurons in area $\mathrm{A}$ exerts an effect on the activity of those in area B. It is sometimes assumed that such a directional influence should be accompanied by a positive time delay (relative phase lead of the activity in area A before that in area B), indicating that A's activity temporally precedes that of B (Sharott et al., 2005; Gregoriou et al., 2009). However, this assumed relationship is not theoretically justified. Furthermore, it has been empirically observed that a dominant directional influence between areas of sensorimotor cortex may be accompanied by either a negative or a positive time delay (Brovelli et al., 2004). Brovelli et al. showed that steady contractions of arm and hand muscles by macaque monkeys performing a visual pattern discrimination task are accompanied by phase synchronization of beta-band (14-30 Hz) Local Field Potentials (LFPs) recorded from somatosensory and motor cortical areas (Brovelli et al., 2004). Directional influence among those areas, as assessed by GC, showed that interareal functional relations are usually asymmetrical. Importantly, the interareal relative phase showed no obvious relation to the directionality determined by the dominant direction of causal influence. Thus, for example, even when $\mathrm{GC}$ indicated that area A exerted a stronger influence on area B than in the reverse direction, suggesting an asymmetric functional relation dominated by the influence from $\mathrm{A}$ to $\mathrm{B}$, it was often the case that area $\mathrm{A}$ lagged behind area B in time (Brovelli et al., 2004).

A similar incongruence between phase difference and GC between PreFrontal Cortex (PFC) and Posterior Parietal Cortex (PPC) in monkeys performing a working memory task was reported by Salazar et al. (Salazar et al., 2012). They observed a dominant parietal-to-frontal beta-band GC influence that 
was opposite to the direction of influence implied by the $2.4-6.5 \mathrm{~ms}$ time lead of PFC before PPC derived from relative phase. The dominant parietalto-frontal direction of GC influence was supported by spike-field coherence analysis, again suggesting that relative phase is not a reliable indicator of directional influence.

In the study of nonlinear dynamics, Anticipated Synchronization (AS) occurs when a unidirectional influence from a dynamical system (A, the sender) to another dynamical system $(\mathrm{B}$, the receiver) is accompanied by a negative phase difference between A and B (Voss, 2000; Voss, 2001a; Voss, 2001b). This counterintuitive synchronization regime can be a stable solution of two dynamical systems coupled in a master-slave (sender-receiver) configuration, provided that the slave also receives a negative delayed self-feedback (Masoller and Zanette, 2001; Ciszak et al., 2003; Ciszak et al., 2004; Kostur et al., 2005; Che et al., 2013). In $\mathrm{AS}$, the receiver's trajectory is able to precede that of the sender by predicting the sender's future behavior. AS has been observed in chaotic systems (Voss, 2000; Pyragas and Pyragiené, 2008; Pyragiené and Pyragas, 2013) and excitable models driven by white noise (Ciszak et al., 2003), and has been experimentally verified in semiconductor lasers (Sivaprakasam et al., 2001; Tang and Liu, 2003) and electronic circuits (Ciszak et al., 2009). It was also shown to occur in 3-neuron microcircuits of noiseless tonic Hodgkin-Huxley models, with delayed self-feedback replaced by a feedback loop mediated by an inhibitory interneuron (Matias et al., 2011). Despite efforts to join concepts of anticipatory behavior and AS dynamics (Stepp and Turvey, 2010; Stephen and Dixon, 2011), biological models of AS, and experimental evidence for it in the brain, have been lacking.

Here we present a dynamical systems model of two cortical regions, coupled with a well-defined directional influence, that displays AS, and compare the model's dynamics in the AS regime to that of LFPs from the cortical data set of Brovelli and coworkers (Brovelli et al., 2004). We report that our model reproduces delay times, as well as coherence and GC spectra, from the cortical data. Our findings provide a theoretical basis for the observed dynamics, in which the primate cortex operates in a dynamical regime where the information flow and relative phase lag have opposite signs. The model further suggests that the local inhibitory interactions in a receiving neuronal population in the cortex will determine whether that population will anticipate or lag behind the sending population. 


\section{Methods}

\section{Modeling synchronization in large-scale systems.}

To simplify the modeling of the asymmetry observed in the Granger causal influences between pairs of areas, we simulated two unidirectionally coupled cortical-like neuronal populations: a sender $(\mathrm{S})$ and a receiver $(\mathrm{R})$, see Fig. $1 \mathrm{1C}$. Each one was composed of 500 neurons (Gollo et al., 2011) described by the Izhikevich model (Izhikevich, 2003):

$$
\begin{aligned}
\frac{d v}{d t} & =0.04 v^{2}+5 v+140-u+\sum_{x} I_{x} \\
\frac{d u}{d t} & =a(b v-u) .
\end{aligned}
$$

In Eqs. 1 1 and 2 is the membrane potential and $u$ the recovery variable which accounts for activation (inactivation) of $\mathrm{K}^{+}\left(\mathrm{Na}^{+}\right)$ionic currents. $I_{x}$ are the currents provided by the interaction with other neurons and external inputs. If $v \geq 30 \mathrm{mV}, v$ is reset to $c$ and $u$ to $u+d$. To account for the natural heterogeneity of neuronal populations, which can exhibit a variety of neuronal dynamics (spiking, bursting, etc. (Izhikevich et al., 2004)), the dimensionless parameters are randomly sampled as follows: $(a, b)=(0.02,0.2)$ and $(c, d)=(-65,8)+(15,-6) \sigma^{2}$ for excitatory neurons (80\% of the population) and $(a, b)=(0.02,0.25)+(0.08,-0.05) \sigma$ and $(c, d)=(-65,2)$ for inhibitory neurons $(20 \%)$, where $\sigma$ is a random variable uniformly distributed on the interval [0,1] (Izhikevich, 2003; Izhikevich et al., 2004). Equations were integrated with the Euler method and a time step of $0.05 \mathrm{~ms}$.

The connections between neurons in each population are assumed to be fast unidirectional excitatory and inhibitory chemical synapses mediated by AMPA and $\mathrm{GABA}_{\mathrm{A}}$. The synaptic currents are given by

$$
I_{x}=g_{x} r_{x}\left(v-V_{x}\right),
$$

where $x=E, I$ (excitatory and inhibitory mediated by AMPA and GABA respectively), $V_{E}=0 \mathrm{mV}, V_{I}=-65 \mathrm{mV}, g_{x}$ is the maximal synaptic conductance and $r_{x}$ is the fraction of bound synaptic receptors whose dynamics is given by:

$$
\tau_{x} \frac{d r_{x}}{d t}=-r_{x}+\sum_{k} \delta\left(t-t_{k}\right)
$$

where the summation over $k$ stands for pre-synaptic spikes at times $t_{k}$. The time decays are $\tau_{E}=5.26 \mathrm{~ms} \tau_{I}=5.6 \mathrm{~ms}$. Each neuron is subject to an independent noisy spike train described by a Poisson distribution with 
rate $R$. The input mimics excitatory synapses (with conductances $g_{E}=$ $0.5 \mathrm{nS}$ ) from $n$ pre-synaptic neurons external to the population, each one spiking with a Poisson rate $R / n$ which, together with a constant external current $I_{c}$, determine the main frequency of mean membrane potential of each population. Unless otherwise stated, we have employed $R=2400 \mathrm{~Hz}$ and $I_{c}=0$. Connectivity within the $\mathrm{S}$ population randomly targets $10 \%$ of the neurons, with excitatory conductances set at $g_{E}^{S}=0.5 \mathrm{nS}$ and inhibitory conductances set at $g_{I}^{S}=4 \mathrm{nS}$.

The $\mathrm{R}$ population is also composed of 400 excitatory and 100 inhibitory neurons, forming the excitatory receiver (ER) and inhibitory receiver (IR) subpopulations (respectively represented by the purple and orange circles in the receiver of Fig. (1C). Neurons in the ER subpopulation receive 40 synapses $\left(g_{E}^{R}=0.5 \mathrm{nS}\right)$ from other neurons of the ER subpopulation, and 10 synapses (with conductance $g_{I}^{R}$ ) from neurons of the IR subpopulation. Neurons in the IR subpopulation receive 40 synapses $\left(g_{E}^{R}=0.5 \mathrm{nS}\right)$ from neurons of the ER subpopulation and 10 synapses $\left(\tilde{\mathrm{g}}_{I}^{R}=4 \mathrm{nS}\right)$ from neurons of the IR subpopulation (Fig. 1C). Note that neurons of the IR supopulation project synapses with different synaptic conductances to neurons in the same subpopulation $\left(\tilde{\mathrm{g}}_{I}^{R}=4 \mathrm{nS}\right)$ and to neurons in the ER subpopulation $\left(g_{I}^{R}\right)$. Subpopulation IR accounts for the inhibitory loop previously reported to be essential for the emergence of AS (Matias et al., 2011). The S and R populations are connected as follows: each neuron of the $\mathrm{R}$ population receives 20 fast synapses (with conductance $g_{E}^{S R}$ ) from random excitatory neurons of the S population.

\section{Characterizing time delay in the model.}

Since the mean membrane potential $V_{x}(x=\mathrm{S}, \mathrm{R})$ of each population (which we assume as a crude approximation of the measured LFP) is noisy, we average within a sliding window of width 5-8 ms to obtain a smoothened signal, from which we can extract the peak times $\left\{t_{i}^{x}\right\}$ (where $i$ indexes the peak). The period of a given population in each cycle is thus $T_{i}^{x} \equiv t_{i+1}^{x}-t_{i}^{x}$. For sufficiently long time series we compute the mean period $T_{x}$ and its variance.

In a similar way we calculate the time delay in each cycle $\tau_{i}=t_{i}^{R}-$ $t_{i}^{S}$ (Fig. 2A). Then we calculate $\tau$ as the mean value of $\tau_{i}$ and $\sigma_{\tau}$ as its variance. In all those calculations we discard the transient time. If $T_{S} \approx$ $T_{R}$ and $\tau$ is independent of the initial conditions, the populations exhibit oscillatory synchronization with a phase-locking regime. We also characterize the regime by the cross-correlation function between the LFPs of the $\mathrm{S}$ and 
R populations (Fig. $2 \mathrm{~B}$ ):

$$
C\left(V_{S}, V_{R}, t\right)=\frac{\left(\sum V_{S}^{i}-\overline{V_{S}}\right)\left(\sum V_{R}^{i+t}-\overline{V_{R}}\right)}{\sqrt{\sum\left(V_{S}^{i}-\overline{V_{S}}\right)^{2} \sum\left(V_{R}^{i}-\overline{V_{R}}\right)^{2}}} .
$$

When directly comparing model results with the experiments, time series obtained from the model had to be downsampled, and the above analysis could not be applied. In that case, the same spectral analysis was applied to both model and data (see below).

\section{Spectral Analysis of LFP and simulation data.}

Coherence, Granger causality and phase difference spectral analysis were calculated following the methodology reported in Brovelli et al. (Brovelli et al., 2004) using the GCCA Matlab toolbox (Seth, 2010). Data were acquired while the monkey was performing a $\mathrm{GO} / \mathrm{NO}-\mathrm{GO}$ visual pattern discrimination task which required it to release (on GO trials) a previously depressed hand lever. Our analysis focuses on 710 trials of the $90-\mathrm{ms}$ period (18 points, $200-\mathrm{Hz}$ sample rate) ending with the visual stimulus onset (wait window). Only correct trials (both GO and NO-GO) were analysed.

The autoregressive modeling method (MVAR) employed by Seth and Brovelli and coworkers (Seth, 2010; Brovelli et al., 2004) to estimate the spectral analysis from the LFP time series requires the ensemble of single-trial time series to be treated as produced from a zero-mean stochastic process. Therefore, we have preprocessed the LFP time series by including detrending (subtraction of best-fitting line), demeaning (subtraction of the ensemble mean) and normalization (division by the temporal standard deviation) of each trial.

It was also necessary to determine an optimal order for the MVAR model. For this purpose we obtained the minimum of the Akaike Information Criterion (AIC) (Akaike, 1974) as a function of model order. The AIC dropped monotonically with increasing model order up to the number of points in a trial minus one (17). We consider that the model order of $10(50 \mathrm{~ms})$ used in (Brovelli et al., 2004) is sufficient to provide good spectral resolution and avoid overparameterization. In fact, we verified the consistency of the results using model orders of 5 and 15 .

For each pair of sites $(l, k)$ we calculated the spectral matrix element $S_{l k}(f)$ (Brovelli et al., 2004; Lütkepohl, 1993), from which the coherence spec$\operatorname{trum} C_{l k}(f)=\left|S_{l k}\right|^{2} /\left[S_{l l}(f) S_{k k}(f)\right]$ and the phase spectrum $\phi_{l k}(f)=\tan ^{-1}\left[\operatorname{Im}\left(S_{l k}\right) / \operatorname{Re}\left(S_{l k}\right)\right]$ were calculated. A peak of $C_{l k}(f)$ indicated synchronized oscillatory activity 
at the peak frequency $f_{\text {peak }}$, with a time delay $\tau_{l k}=\phi_{l k}\left(f_{\text {peak }}\right) /\left(2 \pi f_{\text {peak }}\right)$. Directional influence from site $l$ to site $k$ was assessed via the Granger causality spectrum $I_{l \rightarrow k}(f)$ (Brovelli et al., 2004; Lütkepohl, 1993) (arrows in Fig. 11B).

We also tested our model against published results from a different experiment, where monkeys performed a working memory task while LFP activity from two cortical regions (PFC and PPC) were recorded (Salazar et al., 2012).

\section{Results}

From the experimental data, we have selected four pairs of electrodes for which the two following criteria were satisfied: strongly asymmetric influence inferred by Granger causality and strong coherence. In these cases, both the coherence and Granger causality peaks were at similar frequencies. Those results are represented in Fig. 1 and summarized in Table 1. In all cases the pairs were synchronized in the beta band (around $24 \mathrm{~Hz}$ ).

Whenever a site $l$ strongly and asymmetrically Granger causes $k$, we refer to $l$ as a sender $(\mathrm{S})$ site and $k$ as a receiver $(\mathrm{R})$ site. Intuitively, in these cases one would expect $\mathrm{S}$ to lead $\mathrm{R}$ (i.e. $\tau_{l k}>0$ ), but the counterintuitive result revealed by Table 1 is that there is no consistent relation between GC and $\tau$ (Brovelli et al., 2004; Salazar et al., 2012). Given the complexity of the cortical interactions, several mechanisms could account for this phenomenon. Here we propose a minimal model that explains how asymmetrically coupled neuronal populations can synchronize with either positive or negative time delay.

\section{Delayed and anticipated synchronization in the model}

The asymmetry between $\mathrm{S}$ and $\mathrm{R}$ neuronal populations is structurally builtin in the simulations (Fig. 1TC). Despite the noise and heterogeneity (see Methods), the mean membrane potential of the $S$ and $R$ populations can synchronize with the same main frequency. Depending on the synaptic conductances, the system can exhibit delayed synchronization (DS), with $\tau>0$ (Fig. 2A), or anticipated synchronization (AS), with $\tau<0$ (Fig. 2B). The cross-correlation function $C_{S R}(t)$ corroborates these results, displaying a peak for $t>0$ in the DS regime and for $t<0$ in the AS regime (Fig. 2 $\mathrm{C}$ ).

AS and DS can also be observed in the model at the level of spikes. For each pair of a pre-synaptic neuron in the $\mathrm{S}$ population and a post-synaptic neuron in the ER subpopulation, we have sampled the relative time $t$ between spikes. The histogram of these relative times is again consistent with the previous analyses, with peaks at positive (negative) values for DS (AS) 
(Fig. 2D). Besides, note that in this figure the peak of the spike-time interval probability density is larger at negative values than for positive ones.

Smooth transitions between AS and DS are obtained when the synaptic conductances are varied. Starting from the AS regime, for instance, by increasing the inhibitory synaptic conductance $g_{I}^{R}$ it is possible to continuously decrease the anticipation time, crossing the zero-lag point into the DS regime (Fig. 2E). It is worth highlighting that the mechanism by which AS emerges in the model is clearly not a delay which increases so much that, once it becomes larger than half of the mean period, looks like an anticipation. Note that both the delay times and the anticipation times are always shorter than $T / 2$.

For fixed inhibitory conductances, non-monotonic but continuous transitions AS-DS-AS can also be obtained by increasing the excitatory conductance $g_{E}^{S R}$ (Fig. $2 \mathrm{~F}$ ). Altogether, the phase diagram of the model in the plane of synaptic conductances $\left(g_{E}^{S R}, g_{I}^{R}\right)$ exhibits large regions of AS and DS phases (Fig. 2G), revealing that these collective behaviors are stable. We have found that these results are robust if we employ the membrane potentials of both ER and IR subpopulations as proxies of the slave population LFP (see below), as well as if other model parameters are varied.

We tested the robustness of these results against several variants of the model. For instance, we found that the transition AS-DS still occurs if the relative proportions of the different types of excitatory neurons in the slave population are altered (via a different choice of the dimensionless parameters $c$ and $d$ in the Izhikevich model (Izhikevich, 2006); results not shown). More importantly, since in the mammalian cortex most areas have bi-directional connections (Markov and Kennedy, 2013), we have checked the effects of a bidirectional interaction in the model, by adding 20 fast synapses (with conductance $g_{E}^{R S}$ ) to each excitatory neuron of the M population projected from neurons of the $\mathrm{S}$ population. Increasing $g_{E}^{R S}$ from zero (i.e. the original model), a system in the AS regime $(\tau<0)$ clearly remained in the AS regime until $g_{E}^{R S} \simeq 0.5 g_{E}^{S R}$ (above this value, the networks reached $\tau \simeq 0$, i.e. zero-lag synchronization; results not shown). Therefore, an asymmetry in the synaptic coupling of mutually connected populations is sufficient to yield AS in the model.

\section{Model reproduces experimental coherence and GC spec- tra}

The aim of this section is to verify whether our model can be tuned to reproduce the results reported in (Brovelli et al., 2004) for monkeys performing 
a cognitive GO/NO-GO task. As we have shown in the previous sections, the model already qualitatively reproduces the experimentally observed mismatch between directional influence and phase lag. To reach a quantitative agreement, however, we needed to vary the model parameters. In particular, to tune the peak frequency in the coherence spectrum (24 Hz in Fig. 3), we added a constant current to each neuron $\left(I_{c}=9 \mathrm{pA}\right)$ and adjusted the synaptic conductances $\left(g_{I}^{S}=\tilde{\mathrm{g}}_{I}^{R}=3.2 \mathrm{nS}, g_{E}^{S R}=0.5 \mathrm{nS}\right.$ and $\left.g_{I}^{R}=12.6 \mathrm{nS}\right)$. These modifications also produced noisier time series, as compared to those shown in Fig. 2 A and B, that better mimic the measured LFPs (Fig. 3A). In addition, and for a fair comparison with data, the simulated LFPs were computed by considering the activity of both the ER and IR subpopulations. Moreover, we have down-sampled the model time series to the same rate used in the experiments $(200 \mathrm{~Hz})$, after which simulated data was analyzed exactly like experimental data.

In Fig. 3 we compare simulation results with experimental data from sites 1 and 2 (primary motor and somatosensory cortices respectively, see Fig 11B), which showed a clear unidirectional influence (from 2 to 1 ) and negative time delay. Tuned to AS, the model yielded a coherence spectrum similar to that of the data (Fig. 3B B), particularly in its sharpness around the measured peak frequency. Not surprisingly, the absolute values of the peak in the coherence spectrum for the simulations is larger than for the data, probably reflecting the fact that, differently from our simple model, in the brain one region is also influenced by many other regions. Note, however, that the interpretation of these effects in the experimental results is limited by the bivariate nature of the GC and coherence analyses. Besides the GC spectral analysis, we have also computed the Transfer Entropy (a nonlinear measure of causality detection) by using the HERMES software package (http://hermes.ctb.upm.es/) (Niso et al., 2013) obtaining similar directional influences.

The model also successfully reproduces the main features of the GC spectrum of the data (Fig. $3 \mathrm{C}$ ). A sharp peak was obtained in one direction ( $\mathrm{S}$ $\rightarrow \mathrm{R}$ in the model), whereas the reverse direction showed a weak and flat spectrum. The fact that the frequency of the peak in the GC spectra approximately coincides with the frequency of the peak in the coherence spectra suggests that G-causality is mediated by the coherence oscillations around $24 \mathrm{~Hz}$ (Brovelli et al., 2004).

Results by Brovelli et al. showed positive as well as negative time delays, given an asymetrical GC between two sites (Brovelli et al., 2004). By changing the inhibitory conductance $g_{I}^{S}$, the model is able to reproduce both regimes (Fig. 3D), which correspond to what we refer to as DS and AS, respectively.

In the second dataset, the frequencies of the peaks were around $17 \mathrm{~Hz}$ and 
the average relative phase between PPC and PFC was negative (Salazar et al., 2012).

Our simple model yields similar results with changes in parameters $\left(g_{E}^{S R}=\right.$ $1.0 \mathrm{nS}, g_{I}^{S}=\tilde{\mathrm{g}}_{I}^{R}=7.5 \mathrm{nS}, g_{I}^{R}$ from 6 to $20 \mathrm{nS}, I_{c}=0$ and $\left.R=6000 \mathrm{~Hz}\right)$. In Fig. 3D we summarize the comparison between phase differences observed in the model and in the data.

\section{Discussion}

\section{Neuronal populations can exhibit AS}

Although Voss (Voss, 2000) suggested that AS could explain phenomena such as the delayed induced transition in visually guided movements (Tass et al., 1996), to the best of our knowledge there are no explicit reports of AS in neuronal populations. With rare exceptions (Pyragiené and Pyragas, 2013), previous observations of $\mathrm{AS}$ in theoretical, physical, and biological systems were based on the original framework, which included a negative delayed selffeedback(Voss, 2000; Voss, 2001a; Voss, 2001b; Pyragas and Pyragiené, 2008; Masoller and Zanette, 2001; Kostur et al., 2005; Ciszak et al., 2003; Sivaprakasam et al., 2001; Tang and Liu, 2003; Ciszak et al., 2009; Che et al., 2013). Despite efforts to join concepts of anticipatory behavior and AS dynamics (Stepp and Turvey, 2010; Stephen and Dixon, 2011), direct evidence for it in the brain have not been reported. Here we have shown that substituting the negative delayed selffeedback by a biologically plausible dynamical inhibition can lead to AS in a model of coupled cortical populations. This development opens new perspectives to investigate the existence of the AS regime in other biological systems.

In particular, we have observed the emergence of $\mathrm{AS}$ in populations of neurons from the sensorimotor cortex of a monkey performing sensory discrimination tasks and studied its robustness against external noise, heterogeneity and synapses characteristics. Similarly to what occurs in a 3-neuron motif (Matias et al., 2011), here the anticipation time emerges from the system dynamics, instead of being explicitly hard-wired as a tunable parameter in the dynamical equations (Voss, 2000). Since the time delay depends on the strength of the synapses, AS could be tuned by neuromodulation.

Our simple model shows that very few ingredients are necessary for the emergence of AS between two neuronal populations. Furthermore, when numerical time series are downsampled, subject to noise and analyze in conditions similar to those of cortical LFP data, the model qualitative reproduces the experimental data. In our model, AS yields time lags, as well as coherence and GC spectra, that are in good agreement with experimental results. 


\section{Relative time delay is a poor indicator of directional influence}

It is well known that the correlation between two variables does not necessarily imply that one causes the other. However, there is a tendency in the literature to use the relative phase between synchronized populations to infer which one is the sender region (Sharott et al., 2005; Gregoriou et al., 2009). As we have shown, in our model the leading population does not necessarily drive the lagging population. By definition, in a sender-receiver configuration the direction of information flow is from the sender to the receiver. It means the sender influences the receiver in both AS and DS regimes. As there is no violation of causality, the existence of an AS regime in such systems reveals that the relative time delay does not always indicate the direction of causal relation.

In prior analysis of cortical LFP data (Brovelli et al., 2004), an apparent contradiction was found between the time lag and the GC direction for some pairs of sites (see Table 1). A similar paradox was also reported by Salazar et al. for different cortical regions (Salazar et al., 2012). The apparent contradiction is caused by an assumption that the direction of information flow from one process (A) to another (B) must result in process $\mathrm{B}$ following process $\mathrm{A}$ in time. It is worth mentioning that LFPs might be sensitive to the depth of the recording, which can lead to phase reversal as a function of electrode depth (e.g. (Feenstra and Holsheimer, 1979; Chrobak and Buzsáki, 1998; Alonso and Garcia-Austt, 1987)). Although this could shift some phase delays by $\pi$ radians and possibly confound AS with DS and vice-versa, that would not eliminate the apparent contradiction between phase lag and G-causality. In pairs of brain regions in which DS occurs (as e.g. regions 3 and 1 in Table 1), G-causality and phase lag would not match and would still require an explanation.

The assumption that a receiver B should lag behind a sender A is not justified. Actually, our model of AS not only proves that this intuition can fail but also sets a framework in which an AS regime naturally emerges, reconciling G-causality with a negative phase lag. To the best of our knowledge, this is the first model that exhibits AS between cortical populations. The usefulness of the concept of anticipated synchronization is at least twofold: 1) it provides a concrete (and robust) mechanism by which the apparent contradiction can be resolved and specifically highlights the role that local inhibition could play in the receiver population. 2) given the abundance of synchronization studies in neuronal data, the sheer fact that a novel type of synchronization could occur in the brain seems to be very relevant, offering new possibilities for modeling, data analysis and interpretation. 


\section{Correspondence between dynamical synchronization regime and functional brain state}

In light of the hypothesis that synchronization plays an important role in neural processing and coding (Brette, 2012; Fries, 2005), different dynamical synchronization regimes may be required for flexible communication to occur within a given structural network architecture. For instance, changes in dynamical synchronization state may be necessary for short-term changes in functional brain state related to cognitive processing (Battaglia et al., 2012; Bressler and Kelso, 2001), or long-term changes related to learning. AS may represent such a dynamical state of synchronization, and thus may be able to open new and unexplored perspectives for understanding this type of coding. Our model suggests that even populations with a strongly unidirectional connectivity can exhibit dynamical flexibility. Simply by small changes in the relative weights of excitatory and inhibitory synaptic conductances, a range of synchronization patterns, displaying positive to negative time lags, can be achieved for the same anatomical structure. In fact, recent neurophysiological evidence (Anderson et al., 2013) suggests that top-down attentional influences act to affect the balance of excitation and inhibition in visual cortical area V4.

\section{Perspectives}

Our results are also relevant in light of the growing experimental evidence that the synaptic strength between neurons can undergo spike-timing-dependent plasticity (STDP) (Markram et al., 2011). In the DS regime the sender (presynaptic) neuron fires a spike before the receiver (post-synaptic) neuron, which under STDP rules would facilitate long term potentiation (LTP). On the contrary, in the AS regime the receiver neuron fires a spike before the sender neuron, contributing to long term depression (LTD) (Markram et al., 2011; Bi and Poo, 1998). Since we have shown that a sender-receiver neuronal system can undergo a continuous transition from DS to AS via changes in synaptic conductances, the interplay between these regimes and STDP mechanisms is likely to play a significant role in the process of learning.

Since the model presented here predicts that the AS-DS transition is mediated by synaptic changes, a related question is whether the functional significance of AS and DS regimes (if any) could be unveiled by monitoring G-causality and phase lag during the process of learning a new task. On the conservative side, given the central dependence of phase lag on inhibition in the receiver population, the observation of AS between primary somatosensory and motor areas could be just an epiphenomenon, reflecting 


\begin{tabular}{|c|c|c|c|c|c|c|c|c|c|}
\hline \multirow{2}{*}{\begin{tabular}{|c|} 
Site Pairs \\
$\mathrm{S} \rightarrow \mathrm{R}$ \\
\end{tabular}} & \multicolumn{2}{|c|}{ Peak Coherence } & \multicolumn{4}{|c|}{ Peak Granger Causality } & \multirow{2}{*}{\begin{tabular}{|c|} 
Phase \\
Difference (rad)
\end{tabular}} & \multirow{2}{*}{\multicolumn{2}{|c|}{$\begin{array}{c}\text { Time delay } \\
\tau(\mathrm{ms}) \\
\end{array}$}} \\
\hline & Magnitude & $f_{\text {peak }}(\mathrm{Hz})$ & $\mathrm{S} \rightarrow \mathrm{R}$ & $f_{\text {peak }}(\mathrm{Hz})$ & $\mathrm{R} \rightarrow \mathrm{S}$ & $f_{\text {peak }}(\mathrm{Hz})$ & & & \\
\hline $2 \rightarrow 1$ & 0.3051 & 24 & 0.1944 & 25 & - & - & -1.3166 & -8.73 & (AS) \\
\hline $2 \rightarrow 3$ & 0.4029 & 24 & 0.1547 & 26 & 0.0892 & 25 & -2.1316 & -14.14 & $(\mathrm{AS})$ \\
\hline $2 \rightarrow 4$ & 0.2552 & 24 & 0.1086 & 24 & 0.0265 & 26 & -1.6706 & -11.08 & $(\mathrm{AS})$ \\
\hline $3 \rightarrow 1$ & 0.2546 & 24 & 0.1610 & 24 & $\overline{-}$ & 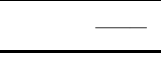 & 0.4637 & 3.08 & $(\mathrm{DS})$ \\
\hline $3 \rightarrow 4$ & 0.7186 & 24 & 0.4203 & 26 & 0.0859 & 28 & 0.3799 & 2.52 & (DS) \\
\hline $4 \rightarrow 1$ & 0.2072 & 24 & 0.0644 & 26 & - & - & -0.4313 & -2.86 & (AS) \\
\hline
\end{tabular}

Table 1: Peak of coherence, Granger causality and time delay between all 6 pairs of sites shown in 1, In each pair, the site which exerts a larger influence on the other is called the sender $(\mathrm{S})$. The other site, which receives the larger influence, is the receiver $(\mathrm{R})$. Positive values of time delay indicates the sender leads the receiver (DS), while negative value indicates the sender lags behind the receiver (AS). A dash (-) indicates that no peak was observed in the Granger Causality spectrum.

strong inhibition at the primary motor cortex in order to prevent movement, as required by the task (Brovelli et al., 2004). Alternatively, the precise timing in the coordination among areas might subserve additional functions, possibly in connection with attention and perceptual coordination.

\section{Acknowledgments}

We thank Drs. Richard Nakamura and Richard Coppola for providing LFP data recorded at the National Institute of Mental Health, and Raúl Vicente and Ernesto Pereda for helpful comments and discussions. Financial support by Coordenação de Aperfeiçoamento de Pessoal de Nível Superior (CAPES), Conselho Nacional de Desenvolvimento Científico e Tecnológico (CNPq), Fundação de Amparo à Ciência e Tecnologia do Estado de Pernambuco (FACEPE) and special programs Programa de Apoio a Núcleos Emergentes (PRONEM) and Programa de Apoio a Núcleos de Excelência (PRONEX) are acknowledged. This work was partially supported by the grant FIS2012-30634 (Intense@cosyp) from MINECO (Spain) and FEDER and Grups Competitius, Comunitat Autonoma de les Illes Balears, Spain. The authors declare no competing financial interests. The funders had no role in study design, data collection and analysis, decision to publish, or preparation of the manuscript. 


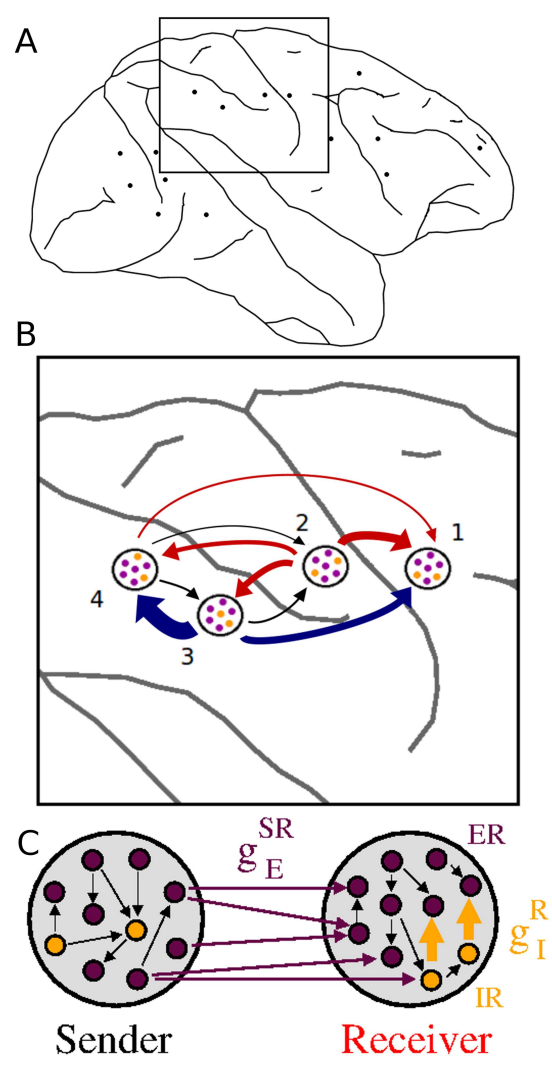

Figure 1: (A,B) Location of recording sites in monkey GE (zoom in the four analyzed electrodes). (B) Sites 1 and 2 are in the primary motor cortex and primary somatosensory cortex respectively. Sites 3 and 4 are in the posterior parietal cortex. Arrows indicate the direction of influence between pairs (Granger causality) and their width are related to the peak of Granger causality shown in Table 1. Colors indicate the sign of time delay between pairs, relative to the influence direction. Blue arrows indicate the sender leads the receiver. Red arrows indicate the receiver leads the sender. (C) Schematic representation of two cortical areas coupled in a sender-receiver (master-slave) configuration. In the model the structural connectivity ensures the direction of influence from the sender to the receiver (mediated by the excitatory synaptic conductance $g_{E}^{S R}$ ). The inhibitory feedback is controlled by the synaptic conductance $g_{I}^{R}$ (see Methods). The effective connectivity may also be accessed by Granger causality measures (see Fig. $3 \mathrm{C}$ ). (A,B modified from Brovelli et al.). 

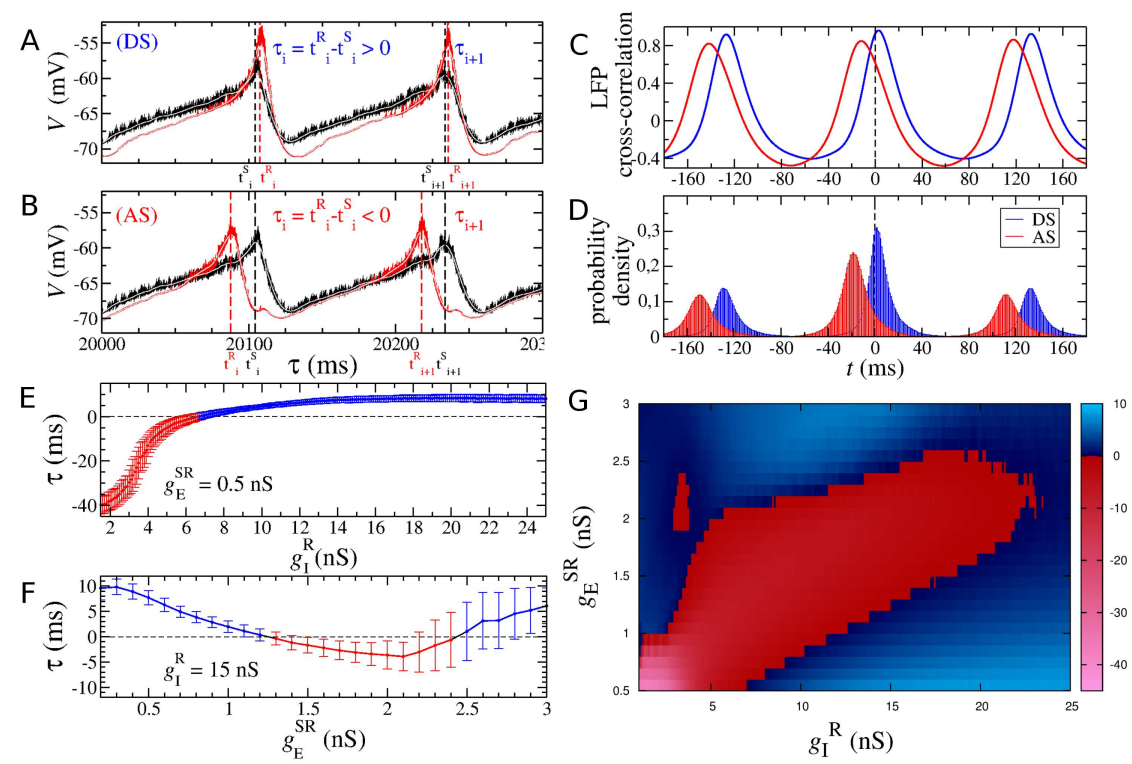

Figure 2: Assessing anticipated and delayed synchronization in a model of sender $(\mathrm{S})$ and receiver $(\mathrm{R})$ populations. Average membrane potential $V$ of $\mathrm{S}$ (black) and $\mathrm{R}$ (red) populations in DS (A) and AS (B) regimes. (C) Cross-correlation between $V_{S}$ and $V_{R}$ for AS (red) and DS (blue) regimes. The time in which the cross-correlation function attains its maximum value is approximately the mean time delay $\tau$ between the $\mathrm{S}$ and $\mathrm{R}$ populations. (D) Normalized histogram of the time delay $\tau^{S R}$ between the spikes of all coupled pairs whose presynaptic neurons are in the $\mathrm{S}$ population and postsynaptic neurons are in the $\mathrm{R}$ population. Time delay $\tau$ as a function of the inhibitory $(\mathrm{E})$ or excitatory $(\mathrm{F})$ synaptic conductances for $g_{E}^{S R}=0.5 \mathrm{nS}$ (E) and $g_{I}^{R}=15 \mathrm{nS}(\mathrm{F})$. (G) Time delay $\tau$ (color coded) in the $\left(g_{I}^{R}, g_{E}^{S R}\right)$ parameter space. 

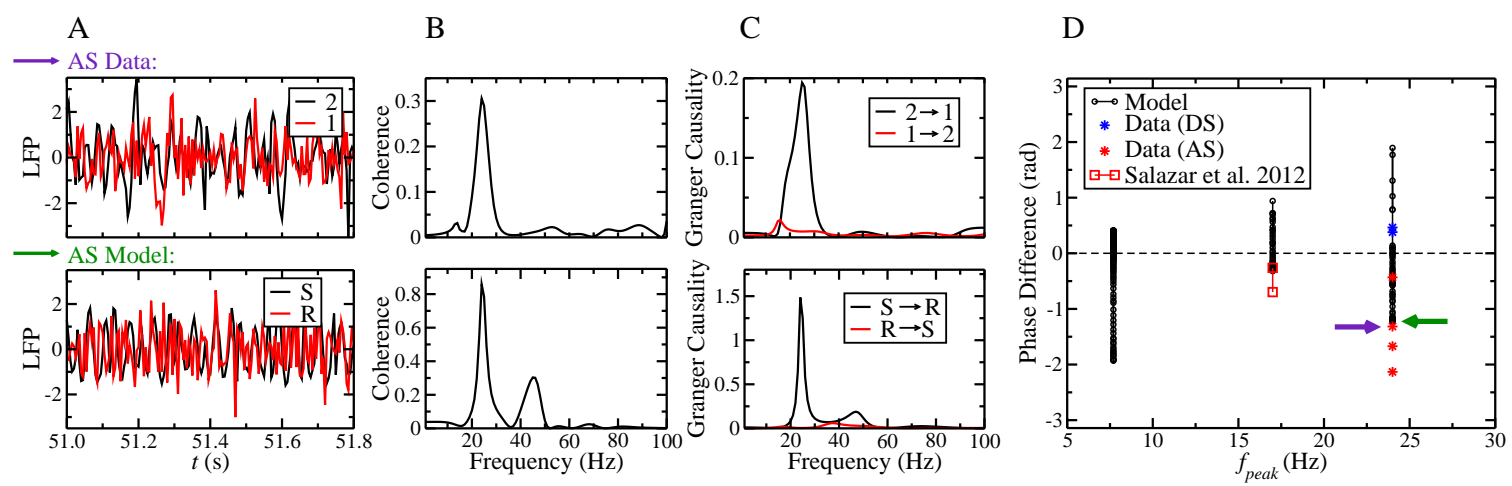

Figure 3: Comparing data from sites 1 and 2 (top) with our model in AS regime (bottom). (A) Measured and simulated LFP time series. (B) Both in data and model the sites are synchronized with main frequency $24 \mathrm{~Hz}$ (peak of the coherence). (C) In data, site 2 Granger causes site 1 (as if site 2 were the sender and site 1 the receiver). However, site 2 lags behind site 1 ( $\tau=$ $-8.7 \mathrm{~ms}$ as shown in Table 1). Similarly, in the model the sender Granger causes the receiver, but lags behind it $(\tau=-8.2 \mathrm{~ms})$. (D) Phase difference between pairs of site as a function of the frequency in which coherence reaches its maximum value $\left(f_{\text {peak }}\right)$. For $f_{\text {peak }}=24 \mathrm{~Hz}$ the model provides phase differences similar to the ones obtained by Brovelli et al., whereas for $f_{\text {peak }}=$ $17 \mathrm{~Hz}$ the model can be compared with the data from Salazar et al.. In this work, posterior parietal cortex Granger causes prefrontal cortex, but prefrontal cortex leads the posterior parietal cortex ( $\tau$ varies from $-2.45 \mathrm{~ms}$ to $-6.53 \mathrm{~ms})$. 


\section{References}

Akaike H (1974) A new look at the statistical model identification. IEEE Trans. Autom. Control 19:716-717.

Alonso A, García-Austt E (1987) Neuronal sources of theta rhythm in the entorhinal cortex of the rat. ii. phase relations between unit discharges and theta field potentials. Rev. Mod. Phys. 67:502-509.

Anderson EB, Mitchell JF, Reynolds J H (2013) Attention-dependent reductions in burstiness and action-potential height in macaque area v4. Nat. Neurosci. 16:1125-1131.

Baccalá LA, Sameshima K (2001) Partial directed coherence: a new concept in neural structure determination. Biological cybernetics 84:463-474.

Banerjee S, Snyder AC, Molholm S, Foxe JJ (2011) Oscillatory alpha-band mechanisms and the deployment of spatial attention to anticipated auditory and visual target locations: Supramodal or sensory-specific control mechanisms? The Journal of Neuroscience 31:9923-9932.

Battaglia D, Witt A, Wolf F, Geisel T (2012) Dynamic effective connectivity of inter-areal brain circuits. PLoS Comput. Biol. 8:e1002438.

Bi GQ, Poo MM (1998) Synaptic modifications in cultured hippocampal neurons: dependence on spike timing, synaptic strength, and postsynaptic cell type. J. Neurosci. 18:10464-72.

Bressler SL, Menon V (2010) Large-scale brain networks in cognition: emerging methods and principles. Trends in Cognitive Sciences 14:277-290.

Bressler SL, Seth AK (2011) Wiener-Granger causality: A well established methodology. NeuroImage 58:323-329.

Bressler S, Kelso J (2001) Cortical coordination dynamics and cognition. Trends in Cognitive Sciences 5:26-36.

Brette R (2012) Computing with neural synchrony. PLoS Comput. Biol. 8:e1002561.

Brovelli A, Ding M, Ledberg A, Chen Y, Nakamura R, Bressler SL (2004) Beta oscillations in a large-scale sensorimotor cortical network: Directional influences revealed by Granger causality. Proc. Natl. Acad. Sci. USA 101:9849-9854. 
Che Y, Li R, Han C, Cui S, Wang J, Wei X, Deng B (2013) Topology identification of uncertain nonlinearly coupled complex networks with delays based on anticipatory synchronization. Chaos 23:013127.

Chrobak JJ, Buzsáki G (1998) Gamma oscillations in the entorhinal cortex of the freely behaving rat. J. Neurosci. 18:388-398.

Ciszak M, Calvo O, Masoller C, Mirasso CR, Toral R (2003) Anticipating the response of excitable systems driven by random forcing. Phys. Rev. Lett. 90:204102.

Ciszak M, Marino F, Toral R, Balle S (2004) Dynamical mechanism of anticipating synchronization in excitable systems. Phys. Rev. Lett. 93:114102.

Ciszak M, Mirasso CR, Toral R, Calvo O (2009) Predict-prevent control method for perturbed excitable systems. Phys. Rev. E 79:046203.

Feenstra B, Holsheimer J (1979) Dipole-like neuronal sources of theta rhythm in dorsal hippocampus, dendate gyrus and cingulate cortex of the urethane-anesthetized rat. Electroencephalography and Clinical Neurophysiology 47:532-538.

Fell J, Axmacher N (2011) The role of phase synchronization in memory processes. Nat. Rev. Neurosci. 12:105-118.

Fries P (2005) A mechanism for cognitive dynamics: neuronal communication through neuronal coherence. Trends in Cognitive Sciences 9:474-480.

Gollo LL, Mirasso CR, Atienza M, Crespo-Garcia M, Cantero JL (2011) Theta band zero-lag long-range cortical synchronization via hippocampal dynamical relaying. PLoS One 6:e17756.

Granger CWJ (1969) Investigating causal relations by econometric models and cross-spectral methods. Econometrica 37:pp. 424-438.

Gregoriou GG, Gotts SJ, Zhou H, R. D (2009) High-frequency, long range coupling between prefrontal and visual cortex during attention. Science 324:1207-1210.

He F, Billings SA, Wei HL, Sarrigiannis PG (2014) A nonlinear causality measure in the frequency domain: Nonlinear partial directed coherence with applications to eeg. Journal of neuroscience methods .

Izhikevich EM (2006) Dynamical Systems in Neuroscience: The Geometry of Excitability and Bursting MIT Press, Cambridge. 
Izhikevich E (2003) Simple model of spiking neurons. IEEE Transaction on Neural Networks 14:1569.

Izhikevich E, Gally J, Edelman G (2004) Spike-timing dynamics of neuronal groups. Cerebral Cortex 14:933-944.

Kostur M, Hänggi P, Talkner P, Mateos JL (2005) Anticipated synchronization in coupled inertial ratchets with time-delayed feedback: A numerical study. Phys. Rev. E 72:036210.

Lobier M, Siebenhühner F, Palva S, Palva JM (2014) Phase transfer entropy: A novel phase-based measure for directed connectivity in networks coupled by oscillatory interactions. NeuroImage 85, Part 2:853 - 872 New Horizons for Neural Oscillations.

Lütkepohl H (1993) Introduction to Multiple Time Series Analysis Springer, Berlin.

Marinazzo D, Liao W, Chen H, Stramaglia S (2011) Nonlinear connectivity by Granger causality. Neuroimage 58:330-338.

Marinazzo D, Pellicoro M, Stramaglia S (2008) Kernel method for nonlinear Granger causality. Physical Review Letters 100:144103.

Markov N, Kennedy H (2013) The importance of being hierarchical. Curr. Opin. Neurobiol. 23:187-294.

Markram H, Gerstner W, Sjöström PJ (2011) A history of spike-timingdependent plasticity. Frontiers in Synaptic Neuroscience 3:1-24.

Marsden JF, Limousin-Dowsey P, Ashby P, Pollak P, Brown P (2001) Subthalamic nucleus, sensorimotor cortex and muscle interrelationships in parkinson's disease. Brain 124:378-388.

Masoller C, Zanette DH (2001) Anticipated synchronization in coupled chaotic maps with delays. Physica A 300:359 - 366.

Matias FS, Carelli PV, Mirasso CR, Copelli M (2011) Anticipated synchronization in a biologically plausible model of neuronal motifs. Phys. Rev. E 84:021922.

Niso G, Bruña R, Pereda E, Gutiérrez R, Bajo R, Maestú F, del-Pozo F (2013) HERMES: towards an integrated toolbox to characterize functional and effective brain connectivity. Neuroinformatics 11:405 - 434. 
Pereda E, Quiroga RQ, Bhattacharya J (2005) Nonlinear multivariate analysis of neurophysiological signals. Progress in neurobiology 77:1-37.

Pikovsky A, Rosenblum M, Kurths J (2001) Synchronization: A Universal Concept in Nonlinear Sciences Cambridge University Press, Cambridge, UK.

Pyragas K, Pyragiené T (2008) Coupling desing for a long-term anticipating synchronization of chaos. Phys. Rev. E 78:046217.

Pyragiené T, Pyragas K (2013) Anticipating spike synchronization in nonidentical chaotic neurons. Nonlinear Dynamics pp. 1-10.

Salazar RF, Dotson NM, Bressler SL, Gray CM (2012) Contentspecific fronto-parietal synchronization during visual working memory. Science 338:1097-1100.

Sauseng P, Klimesch W (2008) What does phase information of oscillatory brain activity tell us about cognitive processes? Neuroscience $\&$ Biobehavioral Reviews 32:1001 - 1013.

Schnitzler A, Gross J (2005) Normal and pathological oscillatory communication in the brain. Nat. Rev. Neurosci. 6:285-296.

Seth A (2010) A matlab toolbox for granger causal connectivity analysis. Journal of Neuroscience Methods 186:262-273.

Sharott A, Peter J Magill PJ, Bolam JP, Brown P (2005) Directional analysis of coherent oscillatory field potentials in the cerebral cortex and basal ganglia of the rat. The Journal of Physiology 562:951-963.

Siegel M, Donner TH, Engel AK (2012) Spectral fingerprints of large-scale neurnal ineteractions. Nat. Rev. Neurosci. 13:121-134.

Singer W (1999) Neuronal synchrony: A versatile code for the definition of relations? Neuron Review 24:49-65.

Sivaprakasam S, Shahverdiev EM, Spencer PS, Shore KA (2001) Experimental demonstration of anticipating synchronization in chaotic semiconductor lasers with optical feedback. Phys. Rev. Lett. 87:154101.

Stephen DG, Dixon JA (2011) Coordinate-independent mapping of structural and functional data by objective relational transformation (ort). Chaos, Solitons and Fractals 44:160-168. 
Stepp N, Turvey MT (2010) On strong anticipation. Cognitive Systems Research 11:148-164.

Tang S, Liu JM (2003) Experimental verification of anticipated and retarded synchronization in chaotic semiconductor lasers. Phys. Rev. Lett. 90:194101.

Tass P, Kurths J, Rosenblum MG, Guasti G, Hefter H (1996) Delay-induced transitions in visually guided movements. Phys. Rev. E 54:R2224-R2227.

Uhlhaas PJ, Pipa G, Lima B, Melloni L, Neuenschwander S, Nikolić D, Singer W (2009) Neural synchrony in cortical networks: history, concept and current status. Front. Integr. Neurosci. 3:17.

Varela F, Lachaux JP, Rodriguez E, Martinerie J (2001) The brainweb: Phase synchronization and large-scale integration. Nat. Rev. Neurosci. 2:229-239.

Vicente R, Wibral M, Lindner M, Pipa G (2011) Transfer entropy?a modelfree measure of effective connectivity for the neurosciences. Journal of computational neuroscience 30:45-67.

Voss HU (2000) Anticipating chaotic synchronization. Phys. Rev. E 61:5115.

Voss HU (2001a) Dynamic long-term anticipation of chaotic states. Phys. Rev. Lett. 87:014102.

Voss HU (2001b) Erratum: Anticipating chaotic synchronization [phys. rev. e 61, 5115 (2000)]. Phys. Rev. E 64:039904.

Wang XJ (2010) Neurophysiological and computational principles of cortical rhythms in cognition. Physiological Reviews 90:1195-1268.

Williams D, Tijssen M, van Bruggen G, Bosch A, Insola A, Lazzaro VD, Mazzone P, Oliviero A, Quartarone A, Speelman H, Brown P (2002) Dopamine-dependent changes in the functional connectivity between basal ganglia and cerebral cortex in humans. Brain 125:1558-1569. 